\title{
Beyond the printed page
}

\author{
The publication of scientific discoveries remained tied to ink and paper for over 300 years, but the rise \\ of the internet over the past few decades has transformed scholarly communication. Just how far this \\ revolution can go depends not just on publishers, but on authors and readers too.
}

Although much has changed since 1665, when the first issue of The Philosophical Transactions of the Royal Society was published, the basic unit of scientific communication is not all that different. The role of an editor at Nature Chemistry is one that Henry Oldenburg, the first editor of Phil. Trans., would probably recognise. But now that most people read journal articles that they have downloaded from the web, rather than pulled down from a shelf, the article itself - not just its delivery - is on the verge of major changes. Some of these are linked to the vision of Tim Berners-Lee and others for the future of the internet - the semantic web $^{1,2}$. In this concept, information is labelled in such a way that computers can understand what it is, rather than just humans, as is generally the case at the moment.

Rather than the HTML version of the article being a narrow reflection of the printed page, it can offer enhancements beyond clicking to bring up figures or references - which simply mimics how people can flick through hardcopies anyway. Enhancements that further enrich articles are already being offered by other publishers, including the Royal Society of Chemistry with Project Prospect ${ }^{3}$. Among other services, this highlights words that are terms in the IUPAC Gold Book and links to their definitions. Beyond simply being an educational tool, this also means that papers on similar concepts are linked together. Apart from the fledgling ChemSpider Journal of Chemistry $y^{4}$, there are few other publishers exploiting the full potential of their online articles. Although the American Chemical Society are testing several interesting and useful innovations on the JACS- $\beta$ website ${ }^{5}$, such as downloadable PowerPoint and ChemDraw files, none of these so far enriches the text of the articles.

Nature Chemistry also offers a number of online enhancements that complement the traditional paper journal. For the large majority of numbered chemical compounds in research articles, a separate compound information web page is available (an example can be found here: http://bit.ly/2WR76) that can be accessed by clicking on the bold compound number - even in the PDF file. These compound

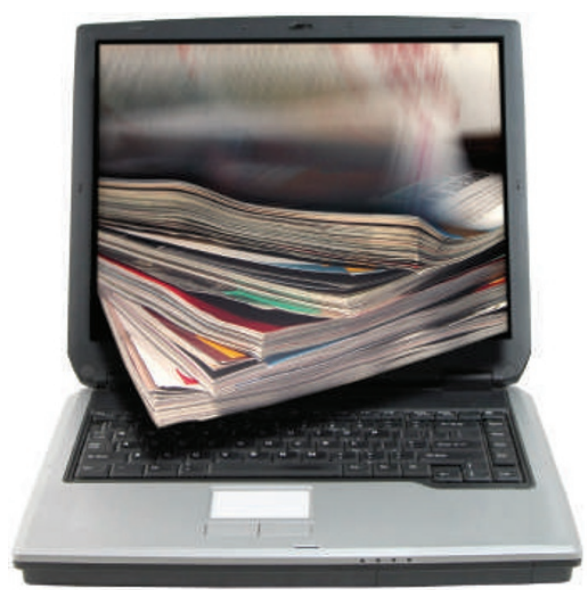

pages include information such as molecular weight and synonyms, as well as an interactive 3D model of the molecular structure that readers can manipulate. Chemical identifiers, such as InChIs ${ }^{6}$ and SMILES $^{7}$ strings, are also included on these pages. These alphanumeric identifiers are machine-readable and can be used in databases and by publishers and chemists to identify and search for chemical compounds. Each numbered chemical compound for any given article is also deposited in the National Institutes of Health PubChem database and a link to the relevant record is included on the compound information page.

Downloadable ChemDraw files for the structures are available for each individual compound, and the compound pages are grouped together on an article-by-article and issue-by-issue basis. A single ChemDraw file containing all of the structures from a particular paper can be downloaded; see, for example, http://bit.ly/Yq2W0. An example of a compound round-up page for an issue can be found here: http://bit.ly/49ggp. All of this gives the reader quick access to much more information, as well as making the individual compounds more visible to the wider chemistry community. As with Supplementary Information, the chemical compound pages are freely available on our website.

A number of enhancements to the text of research articles have also been implemented. A pop-up box containing the chemical structure appears when the mouse cursor hovers over a bold compound number. Other, non-numbered chemical names can be highlighted by clicking the 'Show compounds' link in the right-hand navigation section of an article page. By clicking on a highlighted compound, a pop-up box appears that displays links to free databases where more information about the compound can be found. At present, Nature Chemistry is linking to the PubChem ${ }^{8}$ and ChemSpider ${ }^{9}$ databases. These databases not only display the structure and predicted properties of chemical entities but provide access to other chemical literature by either linking directly to articles or via other databases. There is potential for this process to be used to highlight other families of entities, such as compound classes and reaction types.

Of course, this is only the beginning. Where, and how far, this endeavour can go also depends on the authors and readers of the articles. Individual compound pages already link to compound data such as CIF files for X-ray structures (for example, http://bit.ly/XAbPg). We encourage authors to submit other types of primary data associated with techniques such as NMR spectroscopy or mass spectrometry so that it can be displayed or linked from their papers. Although 'flat' images of experimental data in supplementary material associated with a paper certainly serve an important purpose, the actual data is far more searchable. This is not an effort to detect scientific fraud (data can be faked just as images can), but an opportunity to enrich scientific publications.

How readers will use such resources remains to be seen, but your feedback is welcome in helping us guide our efforts. A blog post that mentions these thoughts is housed on 'The Sceptical Chymist'10 and we encourage you to leave comments.

\footnotetext{
References

1. Berners-Lee, T., Hendler, J. \& Lassila, O. Sci. Am. 284, 35-43 (2001)

2. Berners-Lee, T. \& Hendler, J. Nature 410, 1023-1024 (2001).

3. http://www.rsc.org/Publishing/Journals/ProjectProspect/

4. http://www.chemmantis.com/

http://pubs.acs.org/JACSbeta/

6. Stein, S. E., Heller, S. R. \& Tchekhovskoi, D. in Proc. 2003 Int. Chem. Info. 131-143 (Infonortics, 2003).

Weininger, D., J. Chem. Inf. Comput. Sci. 28, 31-36 (1988).

8. http://pubchem.ncbi.nlm.nih.gov/

9. http:// www.chemspider.com/

10. http://blogs.nature.com/thescepticalchymist/
} 